\title{
MEASUREMENTS OF CUSPAL SLOPE INCLINATION ANGLES IN PALAEOANTHROPOLOGICAL APPLICATIONS
}

\author{
A.V. Gaboutchian ${ }^{\text {a, } * \text {, V.A. Knyaz }}{ }^{\text {b,c }}$, N.A. Leybova ${ }^{d}$ \\ ${ }^{a}$ Moscow State Medical-Stomatological University, 127473 Moscow, Russia -armengaboutchian@mail.ru \\ ${ }^{\mathrm{b}}$ State Research Institute of Aviation System (GosNIIAS), 125319 Moscow, Russia - knyaz@ gosniias.ru \\ ${ }^{c}$ Moscow Institute of Physics and Technology (MIPT), Moscow, Russia \\ ${ }^{\mathrm{d}}$ Institute of Ethnology and Anthropology of Russian Academy of Sciences , 119991, Moscow, Russia - nsuvorova@mail.ru
}

Commission II, WG II/10

KEY WORDS: Photogrammetry, 3D reconstruction, Automation, Non-contact measurements, Anthropology, Odontotomy, Odontometry

\begin{abstract}
:
Tooth crown morphological features, studied in palaeoanthropology, provide valuable information about human evolution and development of civilization. Tooth crown morphology represents biological and historical data of high taxonomical value as it characterizes genetically conditioned tooth relief features averse to substantial changes under environmental factors during lifetime. Palaeoanthropological studies are still based mainly on descriptive techniques and manual measurements of limited number of morphological parameters. Feature evaluation and measurement result analysis are expert-based. Development of new methods and techniques in $3 \mathrm{D}$ imaging creates a background provides for better value of palaeoanthropological data processing, analysis and distribution. The goals of the presented research are to propose new features for automated odontometry and to explore their applicability to paleoanthropological studies. A technique for automated measuring of given morphological tooth parameters needed for anthropological study is developed. It is based on using original photogrammetric system as a teeth $3 \mathrm{D}$ models acquisition device and on a set of algorithms for given tooth parameters estimation.
\end{abstract}

\section{INTRODUCTION}

As an extension to previously reported odontometric techniques used for the development of tooth preparation techniques applied in prosthetic dentistry, we suggested an approach to the measurements of human molars with regard to inclination of their inner and outer cuspal slopes. Our measurement methods are based on a combination of $3 \mathrm{D}$ and $2 \mathrm{D}$ tooth image analysis. $3 \mathrm{D}$ images of teeth are obtained by means of photogrammetry on a specially designed optical system and a dental laboratory laser scanner as well. The images had previously been used for sectioning and carrying out geometric constructions for measurements. Now automated odontometric software is used for the newly introduced applications. Unlike earlier studies, this study is not related to dentistry. The studied samples belong to early and late Middle Ages and are teeth from various regions of the Russian Federation. The goals of our research are to propose new features for automated odontometry and to explore their applicability to paleoanthropological studies

Odontometry has been used in anthropological studies for decades (Zubov, 1973; Hilson, 2003). Classical tooth measurements, carried out on real teeth or their plaster copies (mainly for coronal parts of teeth), include determining two or three sizes in different dimensions by means of calipers (Figure

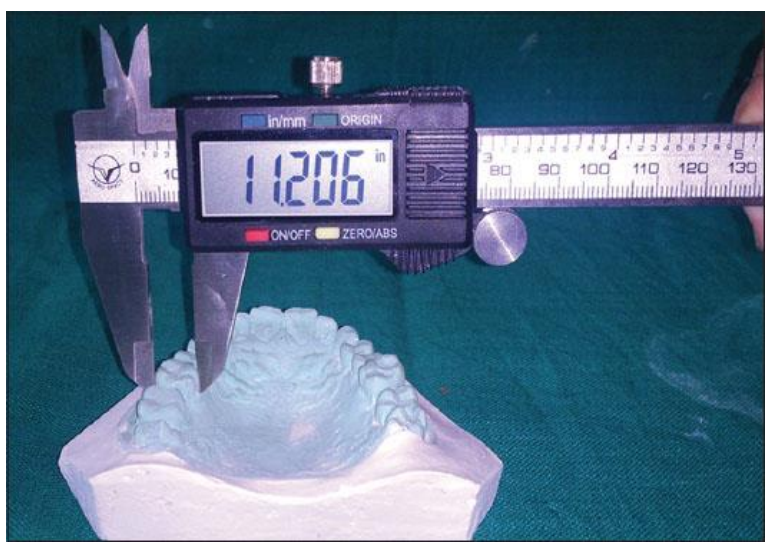

Figure 1. Tooth measurements a on a dental arch plaster model

Certain challenges in the conventional odontometry, such as a lack of appropriate measurement methods which describe tooth shapes, dental morphology specificity, variety in tooth structure, position of teeth in dental arches and their closure etc., determine a higher level of elaboration and relevance of descriptive non-measurement (or non-metric) methods in modern odontology (Figure 2), such as odontoscopy or odontoglyphics (Zubov A., 2006).

\footnotetext{
* Corresponding author.
} 


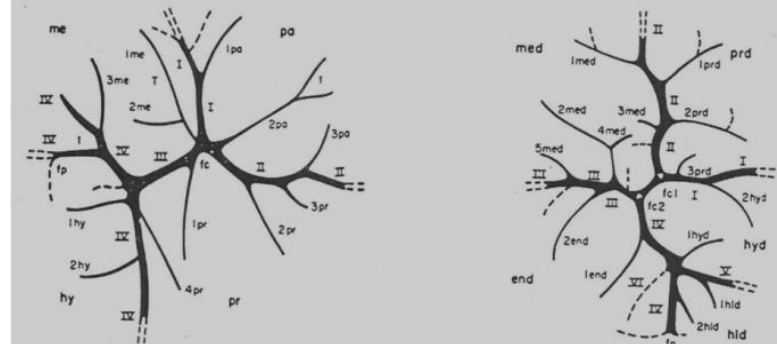

Figure 2. Upper and lower molar fissure patterns.

On the other hand, in recent years there has been an increase in interest to odontology in connection with development of digital 3D techniques. There has been a number of studies related to anthropology or dentistry, including (Naidu and Freer, 2013 a, b; Paredes et al, 2015; Reuschi et al, 2015; Townsend et al, 2012; Zilberman et al, 2003). Our studies have proven effective in describing tooth shape and occlusion (Gaboutchian, 2011), and measurement automation (Knyaz and Gaboutchian, 2016). This encourages us to develop the suggested methods of measurements (Abdoulbekov et al, 2014) not only for applications in dentistry but in anthropology as well.

Being a section of physical anthropology, odontology (from Greek odontos - tooth) interprets tooth crown morphology and its variability as a result of evolutionary process ("dental anthropology" is the widespread term in western literature). Tooth crown morphology represents biological and historical data of high taxonomical value as it characterizes genetically conditioned tooth relief features averse to substantial changes under environmental factors during lifetime. Odontological materials allow cross-group comparison in synchronic and diachronic time scales.

Such evaluations are based on the analysis of numerous autonomous features. Their worldwide distribution has demonstrated that mankind belongs to two major odontological branches - Eastern and Western, which merge on wide territories of Western Siberia, the Urals, Middle Asia and India (Zubov, 1968; 1973). Such a division does not meet traditional classifications into three races - Mongoloid, Caucasoid and Negroid, based on somatological comparisons. The odontological differentiation into two divisions (unlike racial three) indicates of earlier emergence of tooth related traits. What is the reason? Classical racial features, such as colour of skin, hair growth patterns, epicantic fold and etc., have initially developed due to their geographically determined adaptive significance (which is less substantial at the moment). The complex of racial features depicts one of the particular Homo sapiens evolutionary stages which have led, under the influence of various factors, to racial divergence. Odontological features, in turn, are representing other periods of human evolution. They are associated with dissimilar to racial adaptive aspects and, therefore, do not correlate with racial features neither on physiological, nor on historical levels (Zubov, 1973).

The Eastern and Western odontological branches, in their turn, are divided into odontological types - sets of features which are characteristic in differentiating of separate groups. The notion of odontological types is one of the basic in ethnical odontology. The formation of odontological types results from modifications of various odontological features which vary in combinations, in origin and in historical period of formation.
Odontological types are classified in two groups, which are referred to as complexes of reductional and racial features. Reductional complex depicts levels of human tooth apparatus reduction which has been taking place in conjunction with the tendency to skull gracility, noticeable from the Upper Palaeolithic period. Commonly, researchers tend to see reduction as a genetically determined effect. Decrease in skull relief and massiveness was accompanied by bone morphological and structural transformations which have also involved teeth. Modifications determined by reductional complex - including decrease in the number of premolar and molar cusps, lateral incisor reduction, tooth size diminution up to complete reduction (upper lateral incisor, third molar), merging of roots have been developed in various groups unevenly throughout historical periods and variably regarding feature combinations. These circumstances are of high importance in ethnical odontology and palaeoodontology (Zubov, 1968). Odontological types also include features which cannot be related to the process of reduction. The emergence frequency distribution of such features is uneven in diverging populations. The mentioned observations are most likely to be in connection with drift of genes. These features are therefore related to racial and divide the humankind in two major branches - Eastern and Western. Some of these features demonstrate the emergence frequency increase among Mongoloid race and, as a result, are associated with the Eastern branch. Features demonstrating reverse frequency emergence - decreasing to the East and increasing to the West, are related to the Western branch.

Odontologists are in constant search of new phenetic features suitable for differentiating various populations. The majority of such features are descriptive. Such an approach, based on ranking however, is very much dependent on researcher's personal experince. Odontologists have made attempts of broadening the methods of research by involving measurements to improve objectivity in assessments of odontological features, such as shovel-shaped incisors, lingually shifted metacone of upper molars and etc. (Zubov, 1973). Those attempts can be hardly called successive and therefore the mentioned features are not included currently in standard odontological measurement programs. The main reason is in absence of appropriate equipment for fast and precise measurements.

The newly suggested approach provides for qualitative evaluation of tooth crown morthological features. The typical feature of molar cusp slope inclination presented in the article may be potential for high taxonomical significance. In 2000 A.A. Zubov while studying teeth from worldwide known Upper palaeolithic archeological site of Sungir had described the sui generis characteristic of upper molars of an individual from Burial 2 which had displayed high sharpened cuspal tips and innerly inclined cuspal slopes (Zubov, 2000). The assumption testifying of archaic dental system morphology of the individual has not been verified yet in regard to taxonomical reliability due to a lack of precise cuspal slope inclination measurement technique and instrumentaria. Applying of the methods suggested in the article to palaeoanthropological studies is likely to provide for obtaining reliable data on the charactirictic variability during human populations evolutuon on various territories.

Another practical value of the suggested approaches is in providing for precise copying of studied object which are frequently in poor condition due to their age and condition. Creation of virtual collection of unique findings from the 
territory of our country would provide for their potential preservation for studies in the future.

\section{PALEOANTROPOLOGICAL MATERIALS FOR STUDIES}

Along with frequently used plaster copies of teeth for scanning and measurements, we use real teeth as well. In the current study samples of palaeoanthropological origin were used to obtain images. All the 4 samples belong to males who were living in early and late Middle Ages. Two teeth, the first molar and the second molar with an intact coronal part from one individual (the last tooth was extracted from mandible fragment) are of Russian origin. Another two samples (the third molars from different individuals) are from the Northern Caucasus and the Southern Urals, respectively (Fig 3).

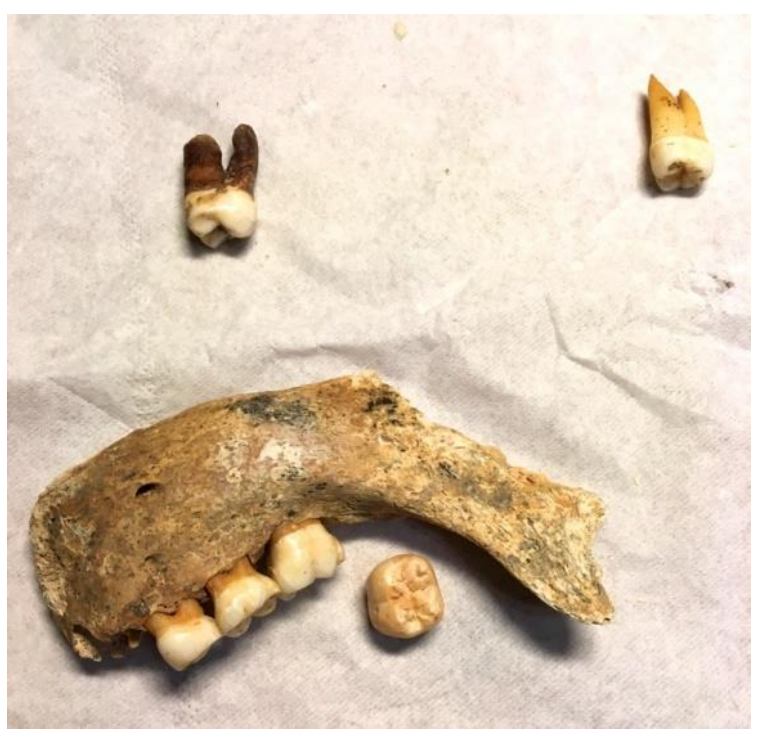

Figure 3. Studied paleoanthropological samples.

Taking into consideration that one of the samples was a fragment of a mandible with two deciduous teeth and a first molar (we were interested study the last one), we immobilised teeth in their sockets by means of light-cured temporary flowable composite resin (Restavrin Tempo, TechnoDent) to provide scanning precision (Figure 4).

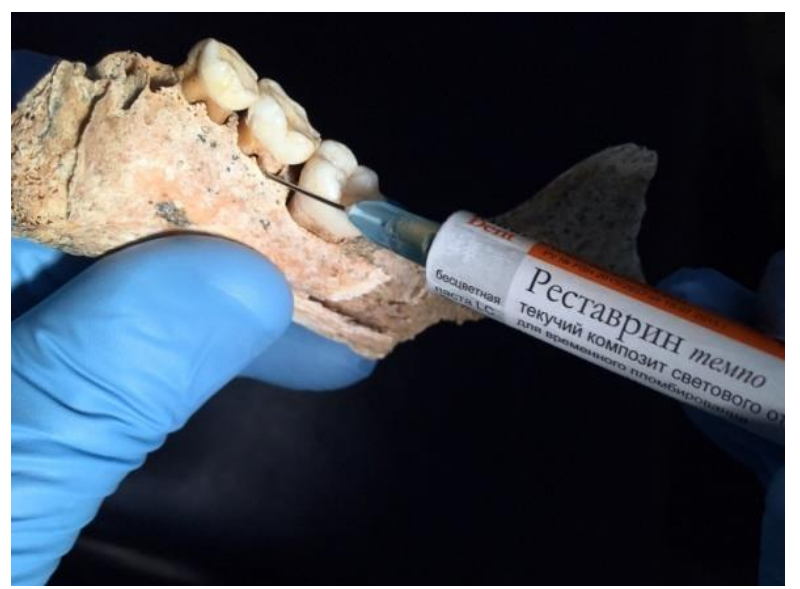

Figure 4. Immobilization of teeth on mandible fragment.
For the same reason samples were covered by 3-D Anti-Glare Spray (Helling) prior to scanning procedure. Images were obtained on originally designed optical photogrammetric system at GosNIIAS and on a laser scanner at dental laboratory.

\section{AUTOMATED 3D MODEL GENERATION AND SOFTWARE}

The images used for our research were obtained by means of original photogrammetric system and professional dental scanner.

\subsection{Photogrammetric system for automated 3D model generation}

A photogrammetric system for automated teeth arch 3D model generation (Figure 5) includes two digital high resolution cameras Basler a641f, high resolution structured light projector and PC-controlled rotation positioning stage. It allows producing high resolution 3D models of teeth arch in fully automated mode.

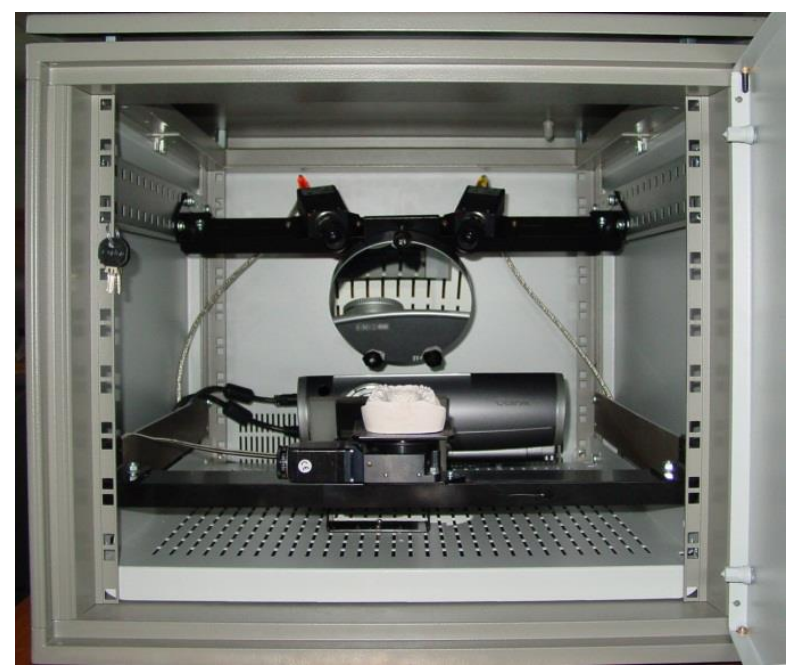

Figure 5. Photogrammetric system for teeth arch 3D model reconstruction

\subsection{Teeth 3D model generation}

The photogrammetric system for teeth arch 3D reconstruction poses the following characteristics:

- High accuracy of 3D reconstruction

- High resolution of tooth 3D model

- Short time for producing teeth arch 3D models

- The possibility of real occlusion registration and reconstruction

- The possibility of occlusion investigation

The photogrammetric system includes two digital IEEE 1394 cameras of high resolution (2 Mpixel), structured light projector with resolution of 1440x1080 pixels and PC-controlled rotation positioning stage. For automated corresponding problem solution coded light is applied providing robust and fast scanning. 
The system is calibrated using the original technique for estimating camera interior and exterior orientation parameters and for determining the parameters of positioning stage rotation axis (Knyaz, 2012). Special calibration test field with coded targets is used for system parameters determination in automated mode. The calibration technique provides residuals of co-linearity conditions for the reference points after least mean square estimation at the level of $0.005 \mathrm{~mm}$. This accuracy is quite adequate for the concerning problem.

For whole teeth arch 3D model generation a set of scans at various plaster model positions is acquired. The number of scans and their orientation are the subject of user choice. All scans are transformed to global coordinate system using the results of rotation axis parameters estimation (Figure 6). This first order scans alignment serves as an initial approximation for accurate scan registration by iterative closest point algorithm.

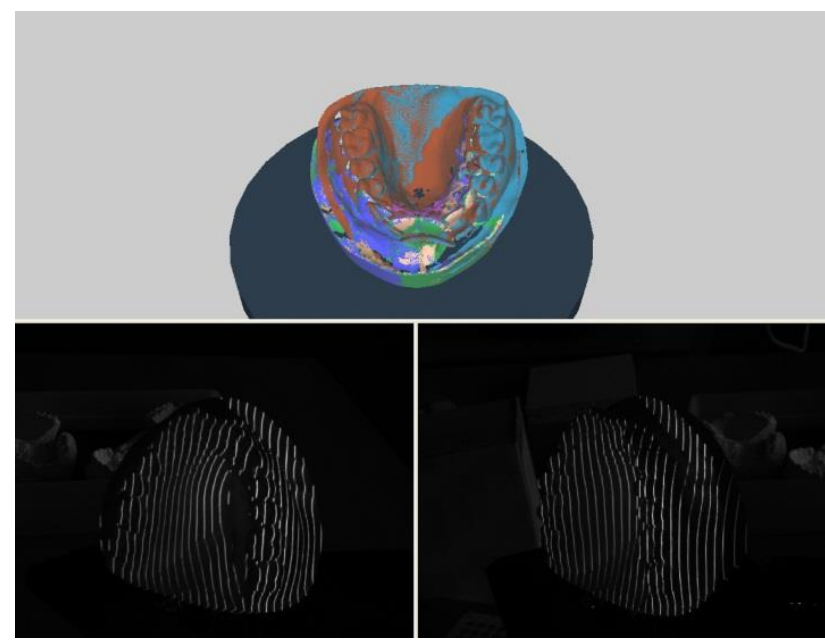

Figure 6.3D reconstruction process

After scan merging a single mesh 3D model is generated using interpolating mesh algorithm.

For acquiring teeth arch 3D model using the described automated procedure it is required about 5 minutes. The resulting 3D model has accuracy of $0.03 \mathrm{~mm}$ and consist of about 1000000 points. Both upper and lower teeth arch 3D models are produced for further occlusion analysis.

The original software for 3D reconstruction and data processing supports the next main functions:

- teeth arch 3D model reconstruction in automated mode

- teeth occlusion registration

- teeth arch segmentation into separate teeth

- teeth shape analysis function (sectioning and section 2D analysis, geometric parameters calculation, shape 3D analysis, etc.)

\subsection{D Model Generation}

The images shown below were obtained on S600 Arti Scanner (ZirkonZahn), which is a fully automated optical structuredlight scanner with 2 high resolution USB 3.0 cameras, providing precision of at least $10 \mu \mathrm{m}$ (courtesy of Tekhnodent dental laboratory, Mytishchi, Russian Federation). The samples were mounted to the device rotation table by means of silicone clamps to provide their safety and stability during the scanning process (Figure 7).

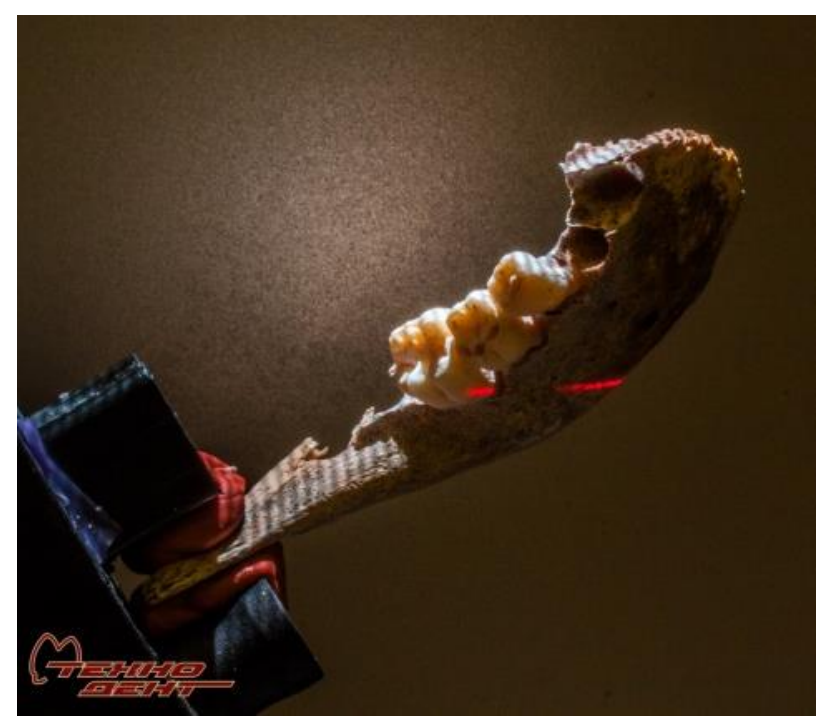

Figure 7. Scanning of anthropological samples.

The results of scanning were acquired as 3D images (.stl) and used for further studies (Figure 8).

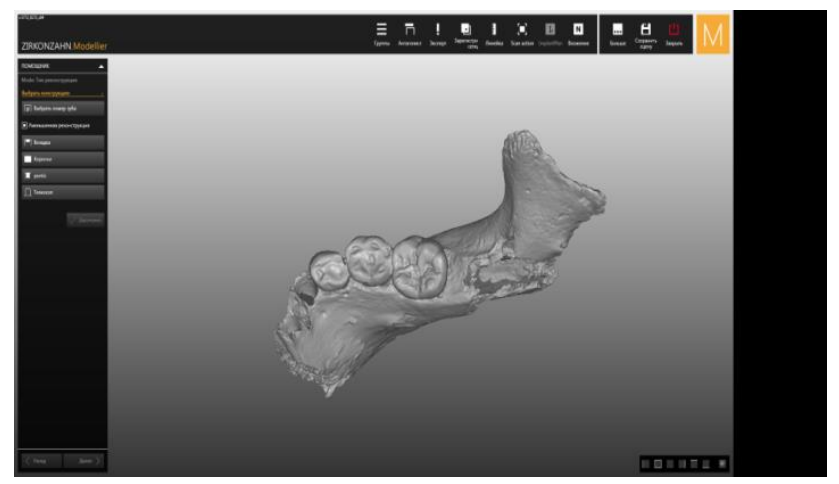

Figure 8. Scanned mandible fragment.

\subsection{Software innovation}

The latest software upgrade, in addition to "manual" mode, has increased the degree of measurement automation that ensures higher speed, precision and objectivity of studies (Knyaz and Gaboutchian, 2016).

\section{ODONTOMETRIC TECHNIQUES}

The method of measurements is based on automated or manual detection of odontometric landmarks on sections of 3D images of teeth. Further geometric constructions and appropriate software development ensures the measurement of teeth, their vestibular and oral cusps and inner and outer cuspal slopes.

\subsection{Odontotomy}

The suggested methods of tooth morphology analysis are based on sectioning of 3D images by series of parallel equally spaced planes. The number of sections and intervals may vary; at the research stage we suggested that equal intervals of $0.1 \mathrm{~mm}$ 
between sections (Figure 9) to ensure study objectivity and equal conditions of obtained data from different teeth.

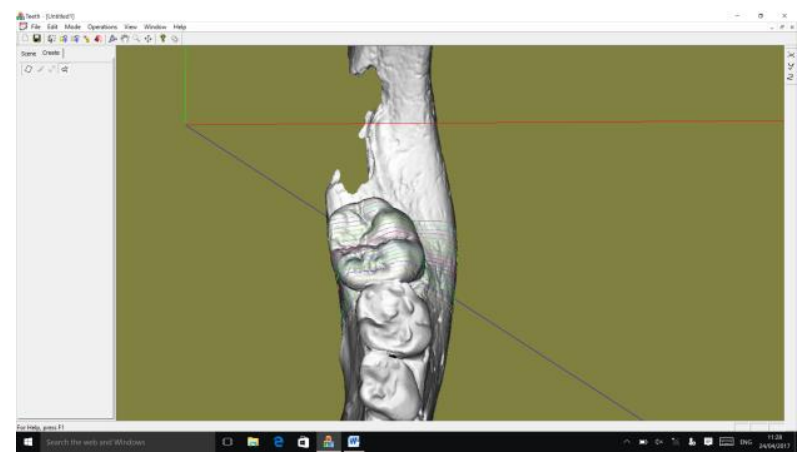

Figure 9. Odontotomy or tooth sectioning.

\subsection{Odontometry}

Without expanding on the initial landmark detection automated methods, as they were described in our previous articles, we are presenting below approaches to cuspal slope inclination measurements. Inner slope inclination measurements technique (Figure 10 a) requires setting unconditional landmarks on premolar and molar contours.

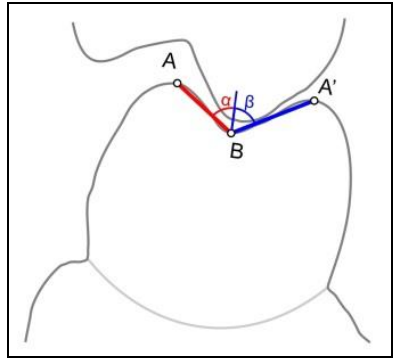

Figure 10 a. Cusp inner slope inclination angles

Subsequently, angle degrees were calculated separately for each cusp slope by means of trigonometric functions (1) (Figure 10 b).

$$
\operatorname{tg} \alpha=x / y
$$

where $\quad \alpha=$ buccal cusp inner slope inclination angle $\mathrm{x}=$ buccal cusp inner slope bucco-lingual width $\mathrm{y}=$ mesio-distal (central) groove depth

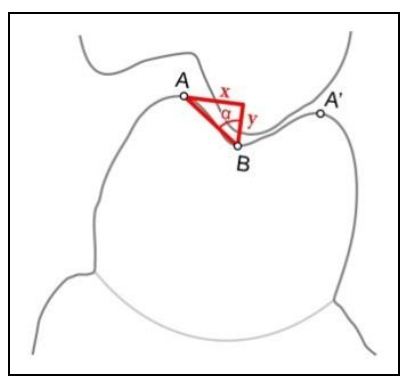

Figure 10 b. Buccal cusp inner slope angle

The angle of tooth inner slope convergence was calculated after cusp inner slope angle measurements (2) (Figure $10 \mathrm{c}$ ).

$$
\gamma=\alpha+\beta
$$

where $\quad \alpha=$ buccal cusp inner slope inclination angle $\beta=$ lingual cusp inner slope inclination angle $\gamma=$ tooth inner slope convergence angle

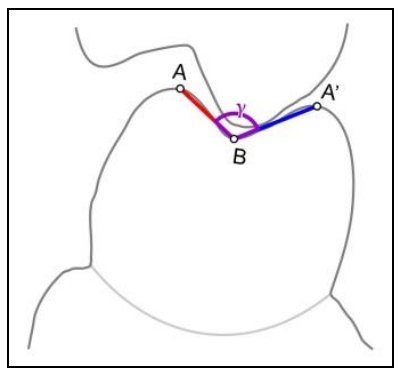

Figure $10 \mathrm{c}$. Tooth inner slope convergence angle

Among odontometric methods published earlier we would like to present in the article a novelty approach to measurements of cuspal outer slope inclination angle (Figure $10 \mathrm{~d}$ ).

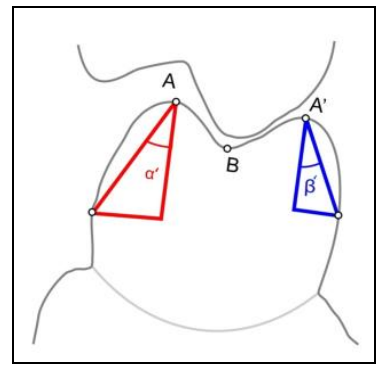

Figure $10 \mathrm{~d}$. Cusp outer slope inclination angles

This gives an opportunity to calculate the angle of cusp inner and outer slope convergence, which helps to evaluate how "sharp" the tooth cusp is (3) (Figure $10 \mathrm{e}$ ).

$$
\delta=\alpha+\alpha
$$

where $\quad \delta=$ cusp slope convergence angle

$\alpha=$ buccal cusp inner slope inclination angle

$\alpha^{\prime}=$ buccal cusp outer slope inclination angle

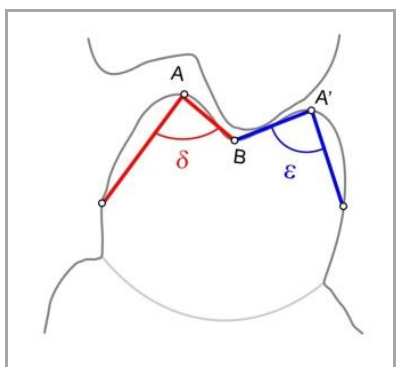

Figure 10 e. Cusp slope convergence angle

\section{MEASUREMENT RESULTS}

Measurements were conducted in automated and manual modes on $2 \mathrm{D}$ tooth contours, obtained through odontotomy (Figure $11)$. 


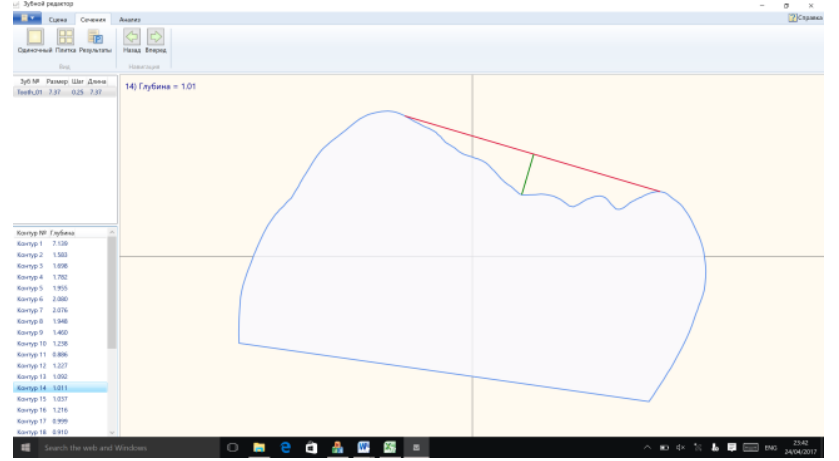

Figure 11. An arbitrary chosen section of the studied tooth.

Angle measurements required geometric constructions which allowed setting of measurement landmarks and defining of parameters required for calculations (Figure 12). Measurements were conducted in accordance to presented odontometric techniques.

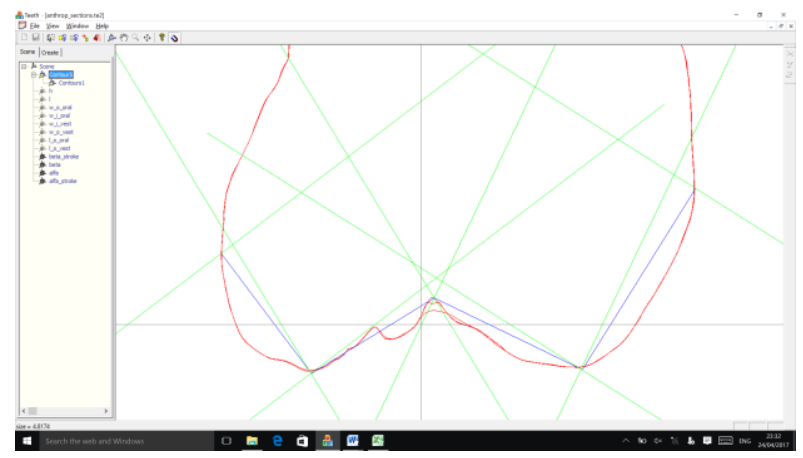

Figure 12. Tooth section with lines defining measured angle sides (blue).

As an example of conducted measurements Table 13 contains results for angle calculations on the section presented above. They demonstrate higher values of inner and outer slope convergence for lower lingual cusps in comparison to buccal cusps on the same section.

\begin{tabular}{|l|c|c|c|c|}
\hline \multirow{2}{*}{$\begin{array}{l}\text { angles, } \\
\text { deg }\end{array}$} & $\alpha^{\prime}$ & $\alpha$ & $\beta$ & $\beta^{\prime}$ \\
\cline { 2 - 5 } & 33.273 & 63.754 & 70.222 & 36.122 \\
\hline \multirow{2}{*}{$\begin{array}{l}\text { angles, } \\
\text { deg }\end{array}$} & $\delta\left(\alpha+\alpha^{\prime}\right)$ & \multicolumn{2}{|c|}{$\gamma(\alpha+\beta)$} & $\varepsilon\left(\beta+\beta^{\prime}\right)$ \\
\cline { 2 - 5 } & 97.027 & \multicolumn{2}{|c|}{133.977} & 106.345 \\
\hline
\end{tabular}

Table 13. Angle measurement results.

Inner slope inclination angles have higher values than for outer slopes on both cusps. The widest angle is presented by $\gamma$ - tooth inner slope convergence angle.

\section{CONCLUSION}

Results of odontoglyphic studies form basic data, while traditional measurements are adjuvant, providing data on tooth sizes in vestibular-oral and mesio-distal dimensions. Thus measurements accepted in anthropology are not effective enough in tooth shape description. In order to expand opportunities of measuring techniques in anthropology and to determine the effectiveness of suggested in comparison to well- tried methods in ethnical anthropological studies, the following research is conducted. Our previous studies for dental applications have proven descriptiveness, objectiveness, precision and high speed of automated odontometry utilising 3D images of teeth, thus providing basis for development of odontometric techniques such as presented cusp slope inclination angle measurements. The obtained results facilitate obtaining important and objective data in anthropological studies which cannot be substituted by applying widely used descriptive methods.

\section{ACKNOWLEDGEMENTS}

The reported study was funded by Russian Foundation for Basic Research (RFBR) according to the research project № 15-0899580 a.

We would like thank colleagues from Tekhnodent dental laboratory (Mytishchi, Russian Federation) for their support in scanning.

\section{REFERENCES}

Abdoulbekov M., Bolshakov G., Gaboutchian A., Isakova T., Kuznetsov O., 2014. The Use of Odontometric Principles in Measurement of Angles of Tooth Cusp Inner Inclines // Dental Forum, No 4, pp. 14-15.

Gaboutchian A.V., 2011. Clinical-experimental study of tooth occlusal surface preparation for prosthetic treatment by fixed prosthesis. PhD thesis synopsis, Moscow, MSMSU.

Hilson S., 2003. Dental Anthropology. Cambridge University Press.

Knyaz V., Gaboutchian A., 2016. Photogrammetry-based Automated Measurements for Tooth Shape and Occlusion Analysis // The International Archives of the Photogrammetry, Remote Sensing and Spatial Information Sciences, Volume XLI-B5, doi:10.5194/sprsarchives-XLI-B5-849-2016.

Mitchell, H.L., Chadwick, R.G., 2008. Challenges of photogrammetric intra-oral tooth measurement. The International Archives of the Photogrammetry, Remote Sensing and Spatial Information Sciences. Vol. XXXVII. Part B5. Beijing.

Knyaz, V. A, 2012: IMAGE-BASED 3D RECONSTRUCTION AND ANALYSIS FOR ORTHODONTIA, Int. Arch. Photogramm. Remote Sens. Spatial Inf. Sci., XXXIX-B3, 585589, doi:10.5194/isprsarchives-XXXIX-B3-585-2012, 2012.

Naidu, D., Freer, T.J. 2013a. The evidence supporting methods of tooth width measurement: Part I.Vernier calipers to stereophotogrammetry. Aust Orthod J. Nov; 29(2):159-63.

Naidu, D., Freer, T.J. 2013b. The evidence supporting methods of tooth width measurement: Part II. Vernier calipers to stereophotogrammetry. Aust Orthod J. 2013 Nov; 29(2):164-9.

Paredes, V., Tarazona, B., Zamora, N., Hernandez, Y., San José, V., 2015. A Comparative Study of Reproducibility, Reliability and Accuracy in Measuring Mesiodistal Tooth Size Using Four Different Methods - 2D Digital, 3D CBCT, 3D Segmented and 3D Intraoral Scanner Method. Issues in Contemporary Orthodontics. http://dx.doi.org/10.5772/59366. 
Reuschi, R.P., Heuer, W., Stiesch, M., Wenzel, D., Dittmer, M.P., 2015. Reliability and validity of measurements on digital study models and plaster models. Eur J Orthodont. doi: http//dx.doi.org/10.1093/ejo/cvj001

Townsend, G., Kanazawa, E., Tokuyama, H., 2012. New Directions in Dental Anthropology: paradigms, methodologies and outcomes. University of Adelaide Press.

Zilberman, O., Huggare, J., Parikakis, K., 2003. Evaluation of the Validity of Tooth Size and Arch Width Measurements Using Conventional and Three-dimensional Virtual Orthodontic Models. Angle Orthod;73:301-306.

Zubov A.A., 1968. Odontology; Methods of anthropological studies / executive editor G.L. Khit'. M.: Nauka

Zubov A.A., 1973. Ethnical odontology. M: Nauka

Zubov A.A., 1979. Ethnical odontology in the USSR / executive editors A.A. Zubov, N.I. Khaldeyeva. M: Nauka.

Zubov A.A., 2000 Morphological study of children`s teeth from the Sunghir burial 2 ( Sunghir 2, Sunghir 3) // Homo Sungerensis. Upper Palaeolitic man: ecological and evolutionary aspects of the investigation. Moscow, Scientific World. P. 256-270.

Zubov A., 2006. Methodological Guidance for Anthropological Analysis of Odontological Materials. ETNO-ONLINE, Moscow. 